\title{
CONCENTRACIÓN Y DISTRIBUCIÓN DE LOS TAMAÑOS DE CIUDADES EN MÉXICO 1940 a 1980
}

\author{
Carlos Brambila \\ y HÉctor SAlazar
}

\section{INTRODUCCIÓN}

EN MÉXICO, COMO EN LA mayoría de los países latinoamericanos, existe un gran interés teórico por entender la relación entre el desarrollo económico y las modalidades del crecimiento urbano. Algunos autores (Hardoy, 1982; Fainstein y Fainstein, 1982) sostienen que en el capitalismo dependiente -sistema de los países latinoamericanos- existe una tendencia a centralizar la actividad industrial y financiera en los grandes centros urbanos; el crecimiento de las ciudades responde así a la funcionalidad económica de concentrar el capital en áreas geográficas en las que el exceso de mano de obra permite mantener los salarios en el máximo nivel de eficiencia.

Por otro lado, el crecimiento urbano en los países dependientes también ha sido explicado en términos del crecimiento natural de las poblaciones rurales que, en el contex to de un panorama agrícola crecientemente deteriorado, provoca altas tasas de emigración ruralurbana y favorece a corto plazo una concentración desmedida de la población rural en los lugares que ofrecen mayores perspectivas de ingreso y bienestar.

En ambas interpretaciones teóricas es posible explicar las fuentes 
del crecimiento urbano de acuerdo a dos factores principales: $a$ ) el creciente deterioro de la actividad agrícola en favor del desarrollo industrial $\mathrm{y}, b$ ) la falta de centros de desarrollo alternativo para la población rural emigrante.

Sin embargo, ninguna de estas teorias ha desarrollado explicaciones coherentes que permitan establecer los límites dentro de los cuales es posible que continúe el proceso de concentración urbana observado, durante los últimos decenios, en la mayoría de los países latinoamericanos. Existe un considerable interés teórico por encontrar las condiciones probables para que la concentración disminuya en favor del crecimiento de ciudades intermedias y pequeñas. Es importante investigar qué condiciones económicas y demográficas favorecen la descentralización del crecimiento poblacional y entender la dinámica del proceso de concentración y desconcentración urbana. Para ello, es necesario analizar la forma en que el crecimiento de las ciudades intermedias afecta a la distribución de tamaños de ciudades en su conjunto.

El objetivo de este trabajo es analizar los cambios experimentados en los niveles de concentración y en los patrones de distribución de los tamaños de ciudades en México, en el período 1940 a 1980.

Examinamos, en primer lugar, las localidades urbanas del país, que en 1970 tenían una población de 15000 o más habitantes, en el período 1940 a 1970 (Unikel, et al., 1978). Después estudiamos el conjunto de las 25 y 37 ciudades mayores del país entre 1940 y $1980 .^{1}$

El procedimiento que hemos seguido para analizar la disposición de acuerdo al tamaño de las ciudades, es la estimación de la expresión lineal de la distribución especial de Pareto, conocida como rango-tamaño, cuya expresión formal es la siguiente:

$$
S_{i}=R_{i}{ }^{-q} S_{1} \circ \log S_{i}=\log S_{1}-q \log R_{i}
$$

en donde $S_{i}$ es el tamaño de población de la ciudad $i ; R_{i}$ es su rango en relación al de la ciudad de mayor tamaño o de primer rango $S_{1}$, y $q$ es un parámetro de proporcionalidad de valor constante igual a la unidad. Esta expresión formal de una distribución tipo Pareto, significa que el tamaño urbano es una función directamente proporcional a su rango correspondiente en un sistema de ciudades, de tal suerte

1 Para este último año hemos utilizado una combinación de datos definitivos dei X Censo General de Población para 25 ciudades y de datos proyectados para 12 ciudades restantes. 
que el producto rango-tamaño de cada una de ellas es constante, $e$ igual al tamaño de la ciudad más grande, es decir:

$$
S_{i} R_{i}=S_{1}
$$

de esta manera, se tendría un sistema de ciudades en el que la segunda ciudad tendría la mitad del tamaño que la primera; la tercera ciudad sería la tercera parte del tamaño de la misma, y así sucesivamente.

Este trabajo es una primera aproximación sistemática sobre el análisis de las distribuciones de tamaños de ciudades en México, en virtud del número de localidades urbanas consideradas entre 1940 y 1970 y la inclusión de 1980; y por el enfoque estadístico adoptado para estimar la expresión formal de la distribución rango-tamaño. ${ }^{2}$

El trabajo está dividido en tres apartados. En los dos primeros analizaremos la concentración y distribución del tamaño de localidades urbanas en México entre 1940 y 1980, y estableceremos una definición de ciudades intermedias, a partir de las tendencias de concentración y desconcentración observadas en los resultados de los coeficientes de Gini y de rango-tamaño registrados. En el tercer apartado sugeriremos algunos elementos teóricos, explicativos de las tendencias de tipo demográfico, económico-spacial y estadístico que podrían investigarse en futuros estudios sobre sistemas de ciudades en México y particularmente en relación a sus posibilidades reales de descentralización.

\section{Distribución DE LA POBLACIÓN URBANA EN MÉXICO}

¿Qué evidencia empírica existe para poder afirmar que la población de México tiende o no a concentrarse en los centros urbanos?

El propósito de este apartado es encontrar una primera respuesta a esta cuestión. Hemos analizado los coeficientes más elementales que permiten estimar el grado de concentración urbana en localida-

2 En el caso de México no se conocen antecedentes de estudios sistemáticos sobre la distribución del tamaño de ciudades, excepto en el caso de los análisis de jerarquía urbana hechos por Unikel, Chiapetto y Garza (1978, pp. 55-60 y 103-114), en los que se privilegia el aspecto de primacía por encima del rango-tamaño. Se conoce sin embargo, un estudio comparativo en 44 países de sus 50 ciudades mayores, cada una de 50 mil habitantes en 1970 o 1971, entre los que se encuentra México, y se demuestra la ventaja del análisis de la regla rango-tamaño en relación al de primacía urbana (véase Rosen \& Resnick, 1980). 
des de diversos tamaños (coeficiente de Gini); posteriormente, estudiamos las estadísticas descriptivas básicas y los coeficientes de la distribución empírica, ajustada a los datos, para observar cómo cambia la tendencia a la concentración urbana en el período de estudio. Finalmente, comentamos dos argumentos teóricos que explican la tendencia a desconcentrar los grupos urbanos del país, tomando como base los procesos demográficos que ocurren en el desarrollo de una población nacional.

\section{Concentración de la población urbana: coeficientes de Gini ${ }^{3}$}

Los censos nos indican que el coeficiente de Gini en 1950 era de 0.416; que experimentaba un aumento en 1960 a 0.495 y que se iniciaba una tendencia creciente en 1970 con un valor de 0.573 . Esta tendencia se observa en la gráfica 1. En ella puede verse cómo las curvas de los años 1960 y 1970 tienden a alejarse paulatinamente de la diagonal principal.

El patrón que sugieren los coeficientes de Gini indica una tendencia de la población a distribuirse, tanto en los centros urbanos principales del país como en localidades menores, que a partir de 1960 , representaron una fuente importante de redistribución de la población de México.

Los cálculos realizados indican una notable concentración de la población urbana en las principales metrópolis del país, pero el hecho importante y sorprendente es la tendencia que muestran las ciudades de "tamaño intermedio" al crecimiento. A partir de 1960 los cálculos de los coeficientes de Gini muestran que uno de los principales factores que permite el nivel constante de concentración es el crecimiento de ciudades de 100000 a 250000 habitantes. Los resultados indican que mientras la proporción de habitantes en localidades grandes y pequeñas, permaneció constante en las ciudades dentro del rango estipulado (100 a 250 mil habitantes), se duplicó porque representaba, en 1950, 9.6\% de la población urbana (en localidades mayores de 10 mil habitantes), ya en 1970, estas ciudades "intermedias" concentran $18 \%$ de la población más urbanizada del país. Esta misma tendencia al crecimiento de ciudades intermedias

3 Los cálculos realizados en este apartado incluyen a todas las localidades del país -rurales y urbanas-y los resultados se muestran en la gráfica 1. 


\section{Gráfica 1}

Curvas de Lorenz para medir la concentración urbana en México: 1940 a 1970

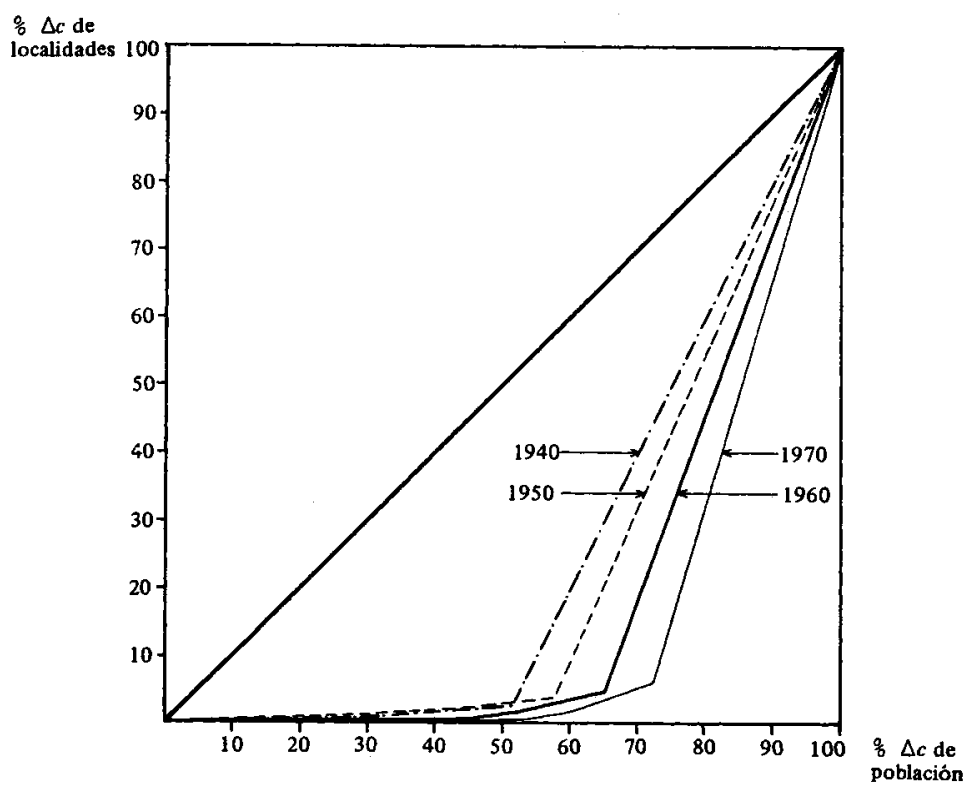

puede observarse aun si se consideran todas las localidades urbanas mayores de 2500 personas. $^{4}$

Por otro lado, los resultados del análisis de concentración urbana, muestran que el número de localidades entre 100000 y 250000 habitantes se duplicó al pasar, de 11 localidades enumeradas a 23, en 1960 y 1970 , respectivamente. De igual manera, la tendencia a la estabilidad del crecimiento en el sistema urbano está relacionado con la multiplicación de ciudades pequeñas de 10000 a 30000 habitantes, que se multiplicaron rápidamente y constituyeron, en todo $\mathrm{el}$ periodo estudiado, un factor importante de desarrollo urbano del país.

La tendencia creciente, observada en el coeficiente de Gini, refleja el proceso continuo de urbanización del país. Sin embargo, consi-

4 Los cálculos de los distintos coeficientes de concentración están disponibles para los lectores interesados, en el Centro de Estudios Demográficos y de Desarrollo Urbano, de El Colegio de México. 
derando que el índice de Gini está afectado por el tamaño de los intervalos utilizados, es importante calcular nuevos coeficientes que incluyan solamente localidades urbanas, con objeto de estimar en qué medida las principales metrópolis del pais tienden a concentrar la mayor parte de la población urbana. Los nuevos cálculos del coeficiente de Gini indican que el nivel de concentración de toda la población urbana del país ha aumentado muy lentamente. En 1940, el coeficiente de Gini (considerando solamente comunidades mayores de 2500 habitantes) era de 0.673 y aumentó, en 1950 , a 0.694 y a 0.716 en 1960 hasta alcanzar 0.739 en 1970 . Es decir, que en un periodo de treinta años la concentración de la población urbana solamente ha aumentado en 0.066 puntos en la escala de Gini. El panorama es aún más estable si se consideran solamente ciudades mayores de 10000 habitantes; los coeficientes de Gini permanecen entonces prácticamente inmutables alrededor de 0.600 durante todo el período de análisis.

Los cálculos de concentración elaborados indican que, aunque las principales metrópolis tienden a tener una mayor proporción de la población urbana del país (entre 30 y $40 \%$ con respecto al total de poblaciones mayores de 2500 ), éste se reduce de $30 \%$ en 1940 a $14 \%$ en 1970 , si se consideran solamente ciudades mayores de 10000 habitantes.

El crecimiento de las ciudades intermedias es significativo lo que, desde el punto de vista teórico contradice las ideas comunes sobre el crecimiento urbano, que proponen que las ciudades principales tienden a atraer crecientemente a la población migrante y que el grado de concentración metropolitana puede llegar a niveles "explosivos".

Para explicar los patrones observados de distribución de la población en el territorio nacional, es importante tener en mente que, hasta la década de los sesenta México fue un país predominantemente rural, con una población dispersa en núcleos menores de 2500 habitantes. Desde 1960, el llamado "desarrollo industrializador" tuvo efectos tangibles en los patrones de redistribución de la población. Sin embargo, el crecimiento astronómico de las metrópolis no es un factor decisivo para la redistribución de la población más dispersa del país, es decir, la que reside en poblaciones menores de 2500 habitantes. El análisis de los datos censales sugiere que el origen de los migrantes a las metrópolis, en las décadas 1940 a 1950 y 1950 a 1960 , sean localidades menores de entre 2500 y 10000 o 20000 habitantes. En cambio el crecimiento de las ciudades de tamaño intermedio que se observa entre 1960 y 1970 probablemente esté 
alimentado por habitantes de localidades rurales más pequeñas. Esta interpretación podría confirmarse por el creciente deterioro de la vida rural y de la seguridad social en el campo.

\section{Distribución de la población por tamaños de ciudades}

Para entender la dinámica del crecimiento de la población urbana en México, es necesario describir estadísticamente la distribución de los tamaños de ciudades con el fin de conocer sus parámetros básicos y mostrar los cambios ocurridos a través del período de interés. Las estadísticas descriptivas de la distribución de tamaños de ciudades en México, se reportan en el cuadro 1 . La distribución en cuestión está altamente sesgada hacia la izquierda y su media crece decenalmente hacia una tasa superior a la del pais de manera que, en promedio, las ciudades crecen $4.6 \%$ aproximadamente cada año. Asimismo, las estadísticas de dispersión muestran cambios notables que reflejan las grandes desproporciones entre las metrópolis y las ciudades intermedias y pequeñas.

Sin embargo, tanto la media como la desviación estándar son estadísticas sensibles a los valores extremos y no muestran el grado de variación de las ciudades más pequeñas. Es más conveniente calcular las estadísticas descriptivas excluyendo de los análisis a las principales metrópolis del país. Las estadísticas correspondientes se muestran en la segunda parte del mismo cuadro.

Un panorama completamente distinto es el que muestran algunas ciudades pequeñas cuya importancia relativa disminuye o, en el mejor de los casos, permanece constante durante las cuatro décadas analizadas. Los resultados del cuadro 2, muestran cómo algunas poblaciones como Piedras Negras y Tapachula, a pesar de pertenecer a las zonas fronterizas del país, disminuyen su tasa de crecimiento y su importancia relativa como centros de atracción a los flujos migratorios.

En síntesis, un análisis detallado del proceso de crecimiento en distintos centros urbanos ha mostrado que la dinámica demográfica de las ciudades intermedias es mucho mayor de lo que generalmente se supone $y$, por lo tanto, requiere de estudios más detallados que permitan distinguir al conjunto de poblaciones urbanas, más susceptibles al crecimiento de las grandes metrópolis y al desarrollo económico en México. Para continuar con el análisis de las ciudades intermedias, que ha sido largamente negado en los trabajos sobre desarrollo urbano de México, es necesario estudiar primero los facto- 
res de proporcionalidad entre el crecimiento de las grandes metrópolis y el de las ciudades intermedias, es decir, elaborar un análisis de la medida en la que el crecimiento de las ciudades más pequeñas tiende a responder o corresponder a la concentración de la población urbana del país en algunos centros preferenciales. En la siguiente sección se

\section{Cuadro 1}

México: Estadísticas descriptivas de la distribución de los tamaños de ciudades, 1900 a 1970

\begin{tabular}{|c|c|c|c|c|c|}
\hline$A \bar{n} o s$ & Media ${ }^{1}$ & $\begin{array}{c}\text { Desviación } \\
\text { estándar }\end{array}$ & $\begin{array}{l}\text { Error }^{1} \\
\text { estándar }\end{array}$ & $\begin{array}{c}\text { Grado de } \\
\text { sesgo en la } \\
\text { distribución }\end{array}$ & $\begin{array}{c}\text { Nümero } \\
\text { de } \\
\text { ciudades }\end{array}$ \\
\hline
\end{tabular}

A. Población de las localidades de 15000 y más habitantes en 1970

\begin{tabular}{rrrrrr}
\hline 1900 & 12655 & 29193 & 2207 & - & 175 \\
1910 & 14607 & 37676 & 2785 & 10.28 & 183 \\
1920 & 16449 & 51070 & 3764 & 11.47 & 184 \\
1930 & 21147 & 79770 & 5896 & 12.21 & 183 \\
1940 & 27425 & 119242 & 8938 & 2.69 & 178 \\
1950 & 45481 & 218079 & 16300 & 2.70 & 179 \\
1960 & 74217 & 371385 & 27528 & 2.53 & 182 \\
1970 & 122134 & 632789 & 47035 & 2.63 & 181
\end{tabular}

\begin{tabular}{rrrrrr}
\multicolumn{7}{c}{ Distribución truncada $^{2}$} \\
1900 & 15932 & 12863 & 1355 & 1.98 & 90 \\
1910 & 19094 & 14934 & 1610 & 1.87 & 86 \\
1920 & 21221 & 16664 & 1874 & 1.93 & 79 \\
1930 & 24313 & 21559 & 2380 & 2.18 & 82 \\
1940 & 26263 & 22426 & 2432 & 2.10 & 85 \\
1950 & 36394 & 32893 & 3273 & 2.32 & 101 \\
1960 & 60596 & 53195 & 5516 & 1.98 & 93 \\
1970 & 114589 & 92334 & 10591 & 1.92 & 76 \\
\hline
\end{tabular}

Fuente: Cálculos elaborados con base en los datos de L. Unikel et al., op. cit., p. 183.

1 En miles.

2 Se excluyen las localidades que caen en el 10 por ciento superior e inferior de la distribución. 


\section{Cuadro 2}

México: Tasas de crecimiento y rangos de ciudades seleccionadas según tamaño de población, 1940 a 1970

\begin{tabular}{|c|c|c|c|c|c|c|c|}
\hline & \multicolumn{3}{|c|}{ Tasas de Crecimiento 1} & \multicolumn{4}{|c|}{ Rango } \\
\hline & 1950 & 1960 & 1970 & 1940 & 1950 & 1960 & 1970 \\
\hline \multicolumn{8}{|c|}{ Metrópolis principales } \\
\hline Cd. México & 6.10 & 5.36 & 5.32 & 1 & 1 & 1 & 1 \\
\hline Guadalajara & 5.10 & 7.05 & 5.32 & 2 & 2 & 2 & 2 \\
\hline Monterrey & 6.20 & 6.80 & 4.49 & 3 & 3 & 3 & 3 \\
\hline Puebla & 4.93 & 2.98 & 5.19 & 4 & 4 & 4 & 5 \\
\hline \multicolumn{8}{|c|}{ Ciudades intermedias } \\
\hline Mexicali & 12.53 & 10.05 & 4.30 & 40 & 16 & 8 & 10 \\
\hline Acapulco & 10.48 & 5.45 & 12.93 & 90 & 46 & 39 & 17 \\
\hline Cuernavaca & 11.06 & 5.82 & 7.72 & 59 & 31 & 26 & 20 \\
\hline Culiacán & 7.98 & 5.52 & 7.05 & 36 & 26 & 24 & 18 \\
\hline \multicolumn{8}{|c|}{ Ciudades pequeñas } \\
\hline Piedras Negras & 5.66 & 4.89 & -0.96 & 53 & 48 & 43 & 71 \\
\hline Tapachula & 6.80 & 3.27 & 3.92 & 56 & 46 & 47 & 52 \\
\hline Comitán & 3.03 & 2.71 & 3.34 & 101 & 110 & 121 & 119 \\
\hline Cortazar & 2.94 & 3.90 & 3.83 & 96 & 107 & 104 & 99 \\
\hline
\end{tabular}

Fuente: Cálculos elaborados con base en los Censos de Población, 1940 a 1980.

1 Calculado con respecto al decenio anterior comor $=\left(P_{t} / P_{o}\right)^{1 / n_{-1}}$ 
analizan estos factores de proporcionalidad en el crecimiento del sistema urbano mexicano.

\section{Distribución rango-tamaño en México}

Los resultados de los cálculos de la distribución de los logaritmos de rangos y tamaños para cada década se muestran gráficamente en las gráficas 2 a 5 . Los resultados muestran en general un buen ajuste entre el rango y el tamaño de las ciudades analizadas. Sin embargo, existen cambios significativos que es importante analizar. Se puede observar en las gráficas respectivas, que el coeficiente de correlación entre los logaritmos de rango y tamaño de las ciudades es bastante alto y tiende a crecer a través del tiempo. La correlación entre rangos y tamaños es de -.926 en 1940 y se incrementa significativamente hasta alcanzar -.984 en 1970 . Este aumento indica una marcada tendencia a la proporcionalidad en el crecimiento de las distintas ciudades del sistema urbano mexicano. Si se considera la alta tasa de primacía del país, la elevada correlación en las variables estudiadas es un claro indicador de que las ciudades intermedias tienden a crecer rápida y proporcionalmente al tamaño de los centros metropolitanos.

Los coeficientes en la regla de rango-tamaño son cercanos a la unidad en todos los años, y se pueden interpretar como un buen ajuste a la distribución de tamaños de las ciudades, considerando los estándares internacionales. Estos indicadores se pueden observar al final de las gráficas 2 a 5 y muestran cómo los coeficientes decrecen lentamente entre 1940 y 1960 , pasando de -1.064 en 1940 a -1.037 en 1960, pero aumentan rápidamente en 1970 hasta alcanzar nuevamente un nivel menor a la concentración observada en 1940, es decir, un coeficiente de -1.079 . Claramente, los resultados indican una tendencia a la proporcionalidad de las ciudades.

Estas variaciones en los coeficientes de la regla de rango-tamaño pueden explicarse por la fuerte tendencia a concentrar las tasas anteriores a 1960 y 1970 y muestran cómo las ciudades intermedias incrementan su ritmo de crecimiento notablemente durante las últimas dos décadas y aumentan su importancia relativa (rangos) dentro del sistema urbano del país. Obviamente, el crecimiento de estas ciudades está estrechamente relacionado con el tipo de desarrollo económico del país.

Hasta aquí hemos analizado el alto grado de variación que muestran las ciudades intermedias y pequeñas a través del periodo 
propuesto. Sin embargo, una pregunta que falta responder es qué proporcionalidad guarda el crecimiento de las ciudades intermedias, con respecto a la dinámica del crecimiento en los centros metropolitanos. Esta pregunta es importante ya que en la literatura sobre el desarrollo urbano de los países, es ampliamente reconocido que el crecimiento de las ciudades más pequeñas está en función del de los principales centros urbanos. Para analizar la proporcionalidad entre ciudades pequeñas, intermedias y grandes es posibie ajustar una distribución empírica que permita cuantificar el nivel en el que la distribución de los tamaños de ciudades se desvía de un patrón de proporcionalidad perfecto. Esta distribución empírica es la regla de rango-tamaño, descrita con anterioridad.

Respecto a esto último es de esperar que el importante crecimiento de las grandes ciudades, durante esta década (1960-1970), no minimice el de las ciudades intermedias que, como se sugirió anteriormente, crecen a través de otros flujos migratorios. Este problema se analizará en el siguiente apartado.

Antes de estudiar la dinámica del crecimiento de las ciudades intermedias, es importante notar una característica importante en las gráficas 2 a 5 , con respecto al ajuste de la línea de regresión de la relación analizada. Como se puede observar en las figuras correspondientes, el ajuste de las líneas de regresión es bastante exacto.

Sin embargo, es posible observar en todas las gráficas una concavidad positiva en las curvas y una parte en la cola de la distribución que baja rápidamente después de cruzar la línea de regresión. A este respecto, John B. Parr (1976) demuestra que, tanto la concavidad con respecto al origen como la cola de la distribución, indican que se trata de una forma particular de la distribución log-normal, cuya estimación se puede hacer con dos o tres parámetros. El hecho de que los tamaños de ciudades en México tengan una distribución lognormal es importante porque permite identificar un conjunto de ciudades que efectivamente muestren un crecimiento proporcional entre si. A la vez, la identificación de ciertas ciudades que crecen homogéneamente permite definir empíricamente un sistema urbano cuya dinámica demográfica depende, en gran medida, del nivel de las principales metrópolis. Las implicaciones de estos análisis se discutirán en el siguiente apartado. 
Grática 2

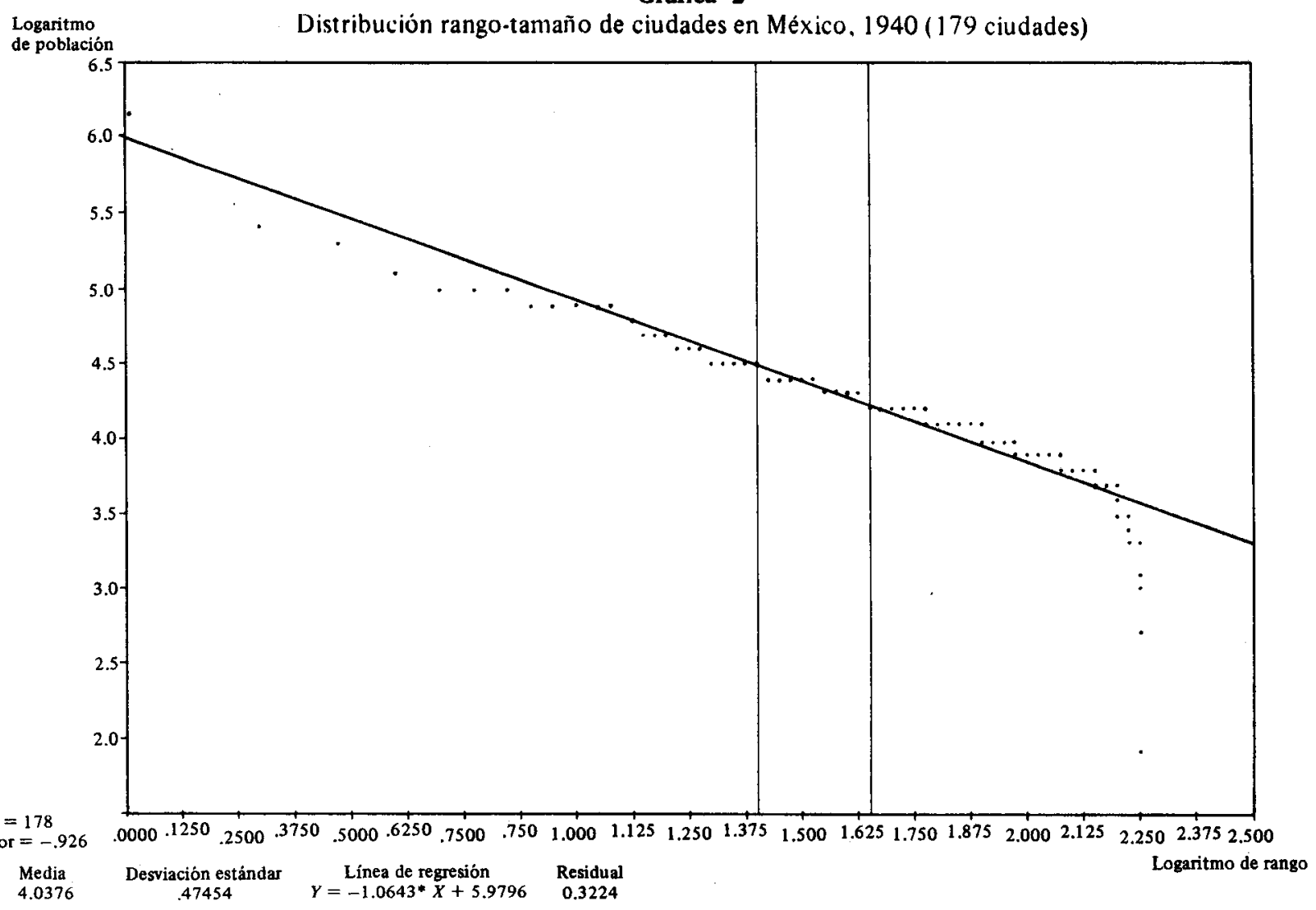


Gráfica 3

Distribución rango-tamaño de ciudades en México, 1950 ( 179 ciudades)

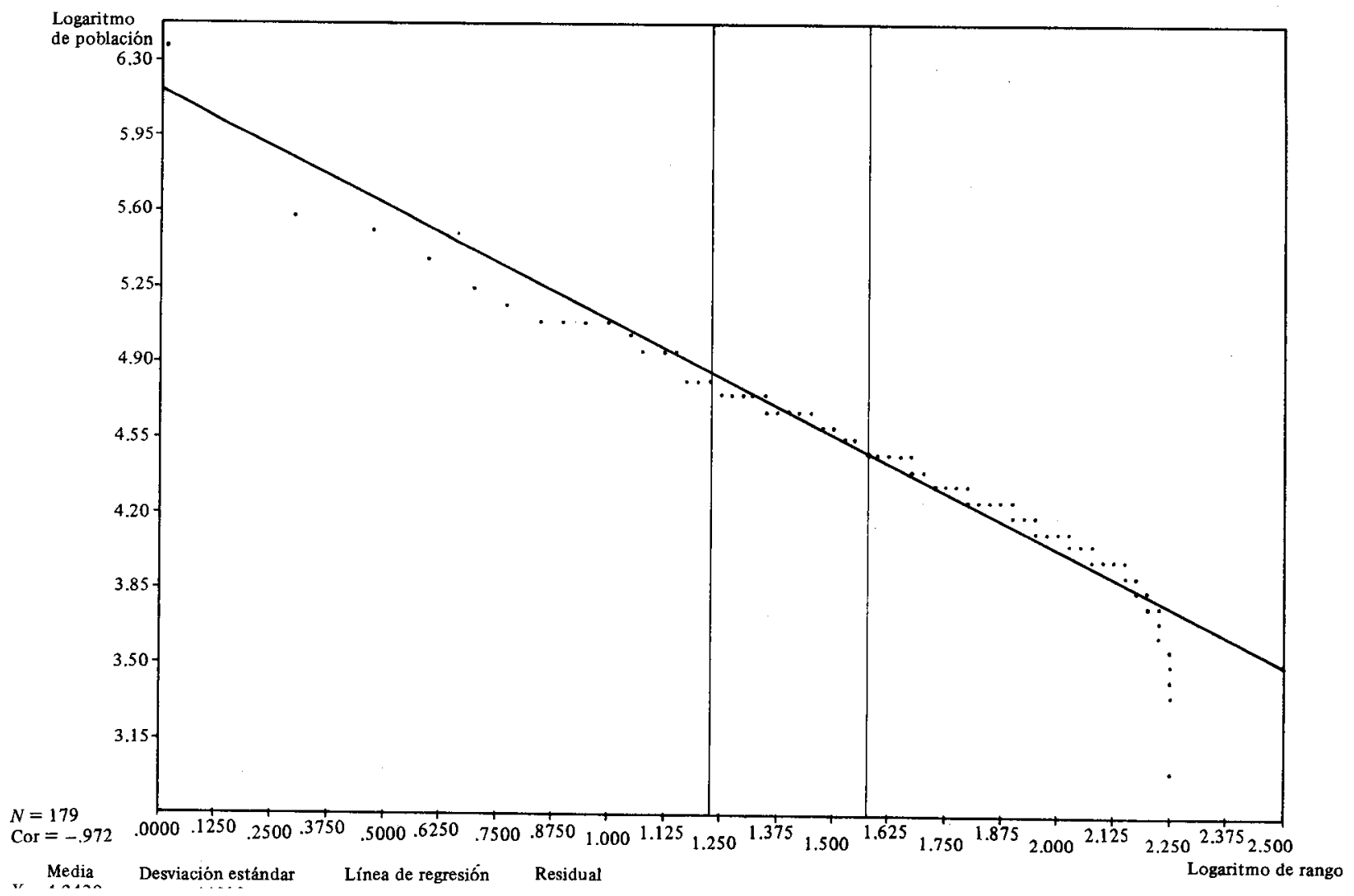




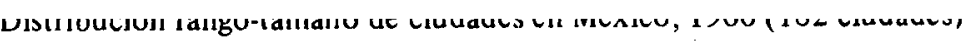

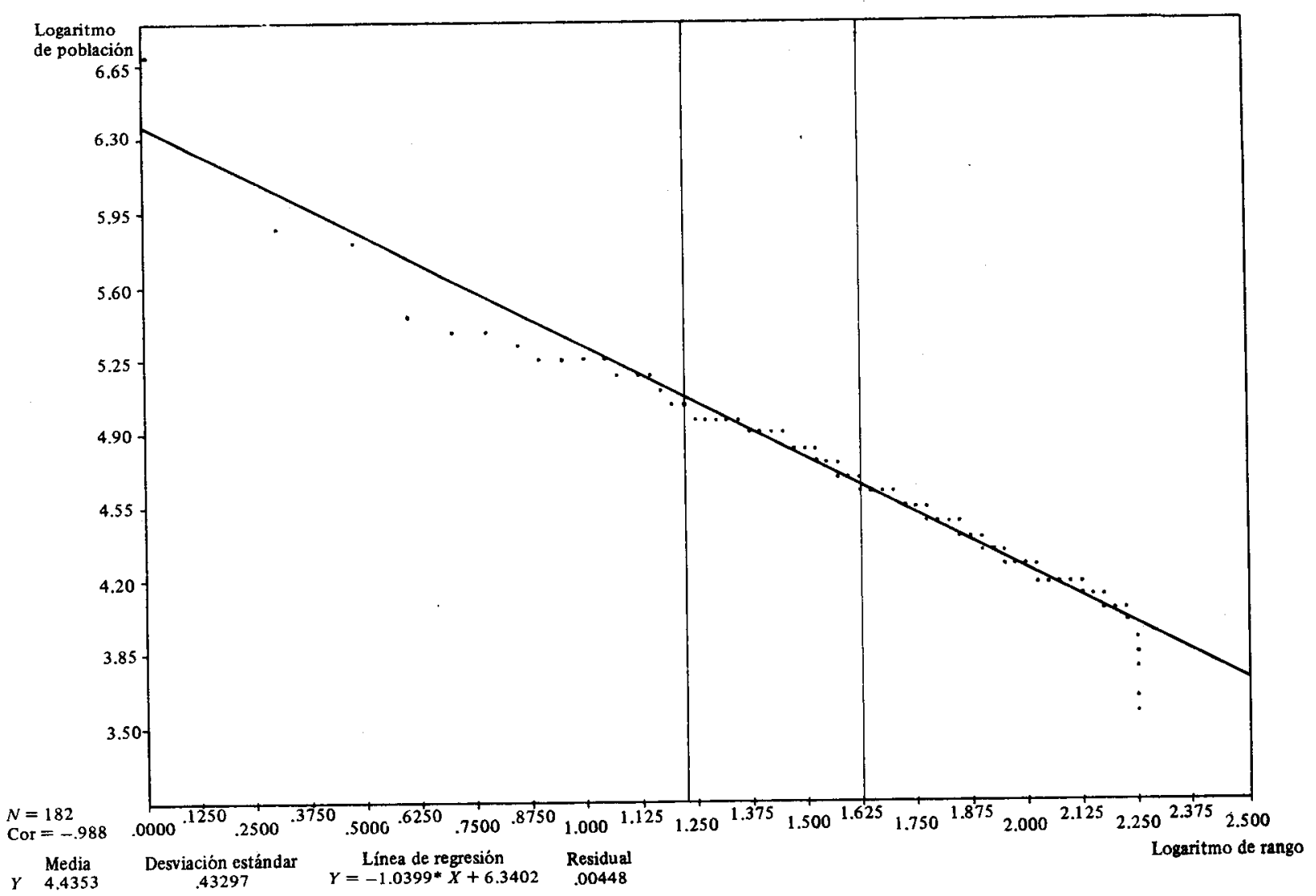


<smiles>C1=CC=C1</smiles> 


\section{Crecimiento de ciudades intermedias en México}

El análisis de las tendencias rango-tamaño de las 178-181 localidades urbanas del país - de 15000 y más habitantes- para 1940 y 1970 , mostró que la regla rango-tamaño es consistente, especialmente para aquellas localidades que van desde el primer rango hasta los rangos 18, e incluso hasta el 42.

Por esta razón, consideramos conveniente analizar el comportamiento de la regla rango-tamaño para un promedio de 30 ciudades. Así, decidimos tomar las 25 ciudades mayores en el período 1940 y 1970 (número intermedio entre el rango 18 y 30) y las 37 ciudades mayores del período 1940 y 1980 (número intermedio entre el rango 30 y 42) a las que llamaremos intermedias por razones que justificaremos más adelante. El resto de las localidades urbanas parecen seguir otro patrón en cuanto a la distribución de sus tamaños, conocido como log-normal (Parr, 1976), por lo que quedan descartadas en este análisis.

\section{Rango-tamaño en las 25 ciudades de mayor tamaño}

Dentro de este grupo de ciudades, las desviaciones estándar de los tamaños y de los rangos no disminuyen considerablemente en relación a las estadísticas calculadas en el caso de las 178 a 181 localidades urbanas entre 1940 y 1970 (véanse las gráficas 6, 7, 8 y 9). La elasticidad entre tamaño y rango se mantienen prácticamente constante alrededor de la unidad y fracciones que no alcanzan valores decimales. Los coeficientes de correlación entre tamaño y rango para los cuatro años considerados se mantienen considerablemente altos con valores no menores de 0.95 .

Estas estimaciones confirman la validez de la regla rango-tamaño entre las 25 ciudades más grandes, a pesar del efecto distorsionador generado por el tamaño de tres áreas urbanas del país: (México, Guadalajara y Monterrey). Puede notarse que las disminuciones en los valores de las desviaciones estándar no indujeron prácticamente variaciones en los valores de la elasticidad rango-tamaño y en su correlación entre los años 1940 a 1970.

Sin embargo, este comportamiento rango-tamaño, verificado para las 25 ciudades más grandes, no es suficiente para sugerir una situación vigente de desconcentración urbana en el país, a juzgar por las pocas variaciones observadas. En efecto, el pequeño alejamiento 
Gráfica 6

Distribución rango-tamaño de ciudades en México, 1940 (25 ciudades)

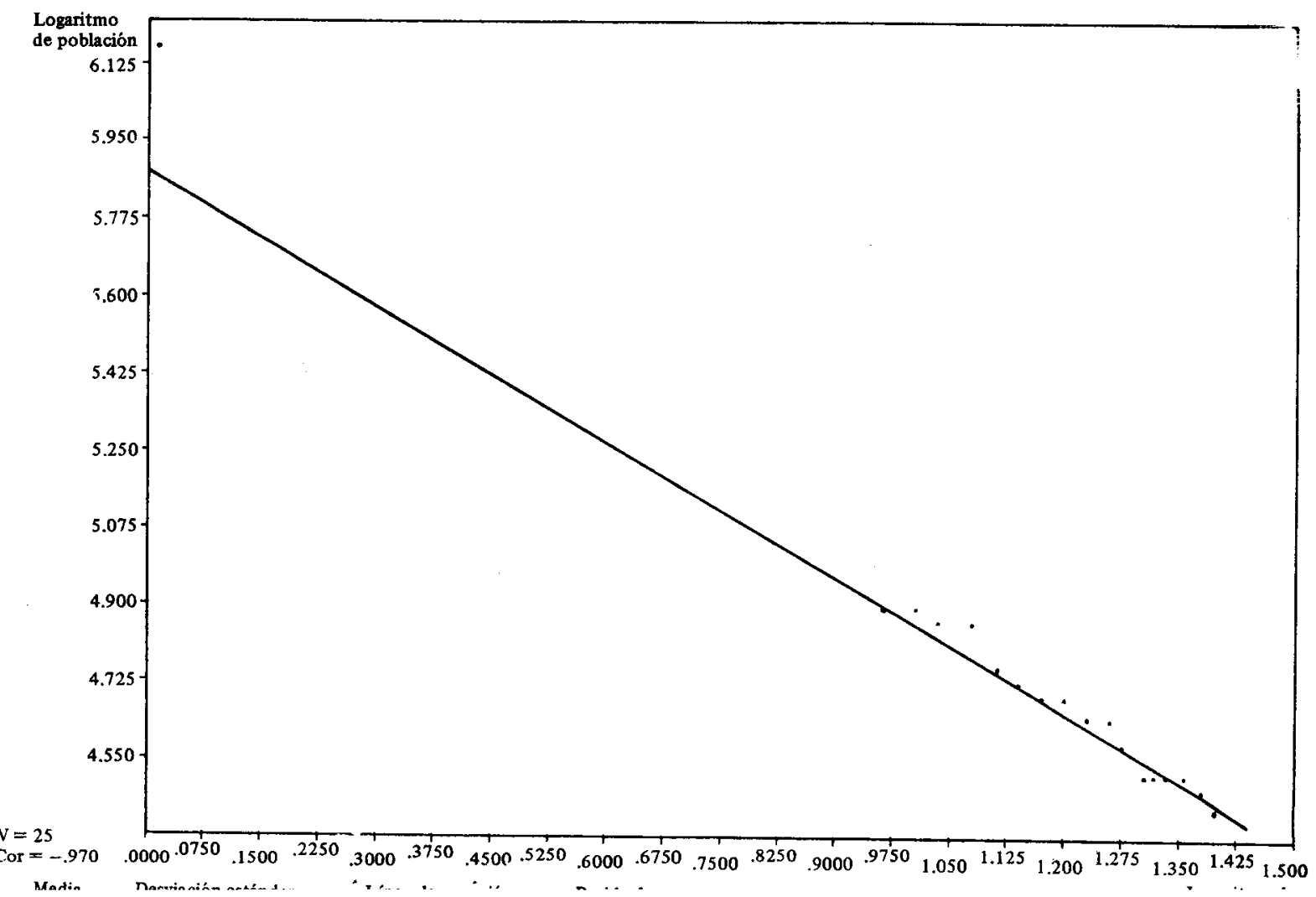




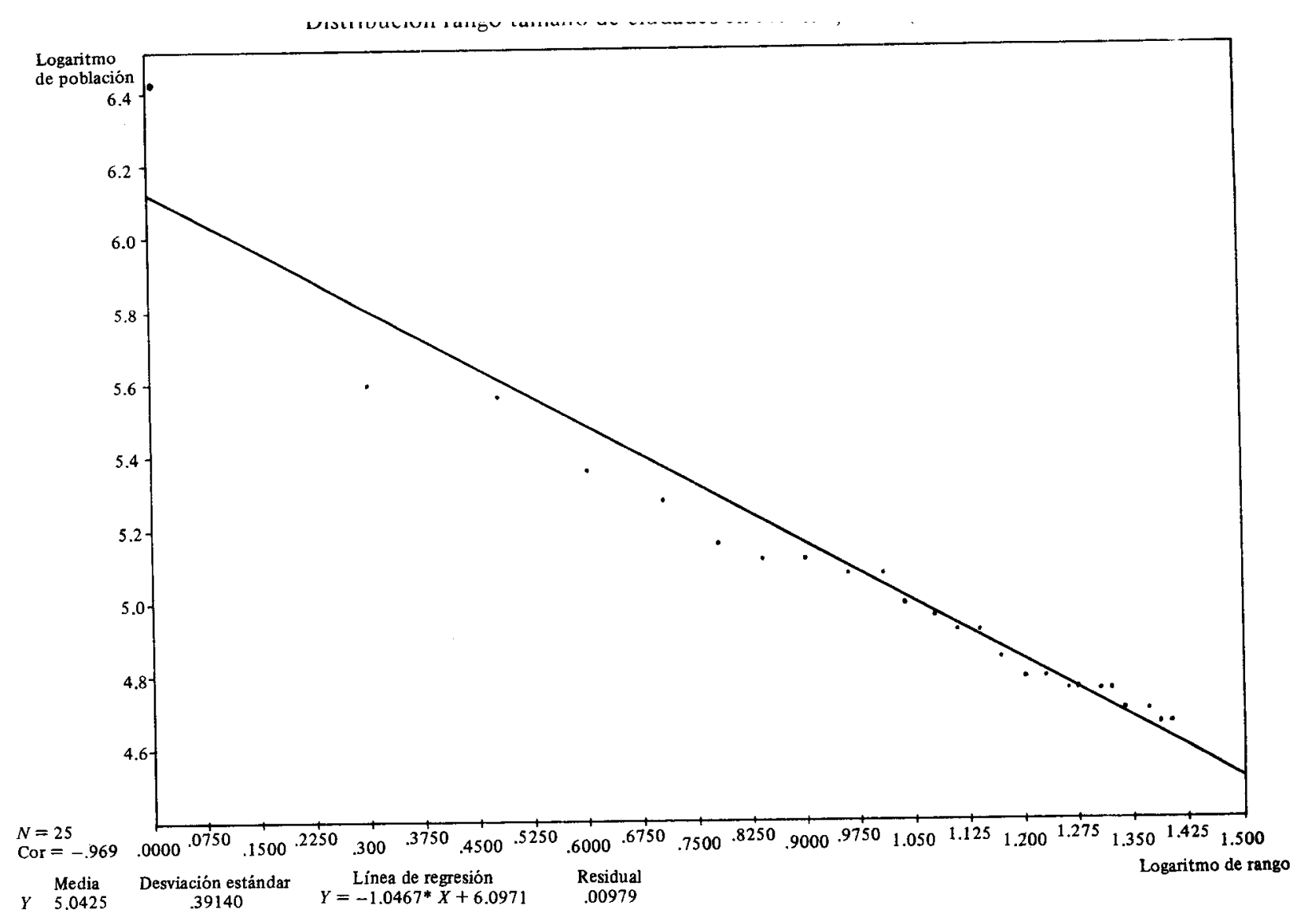




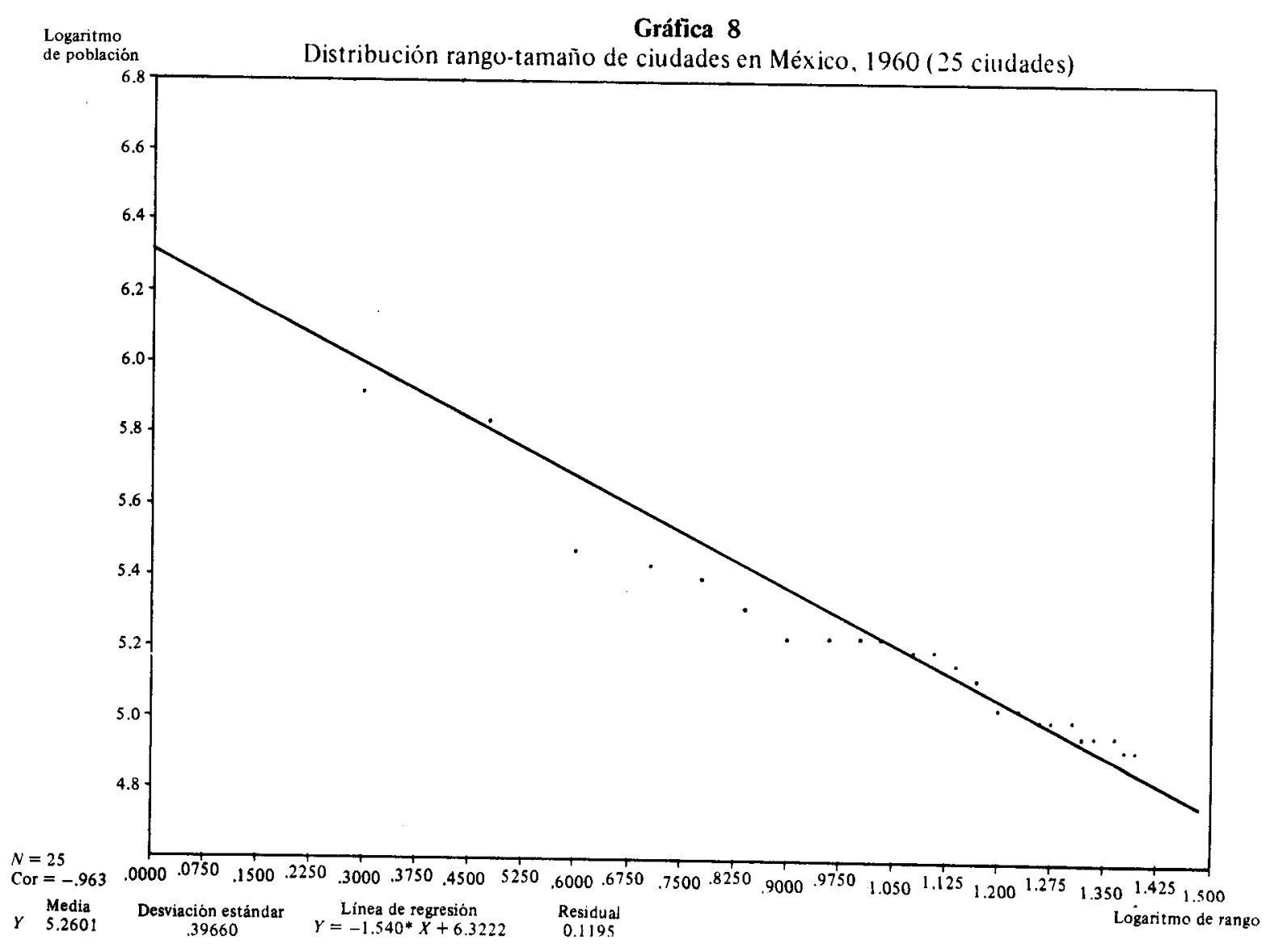




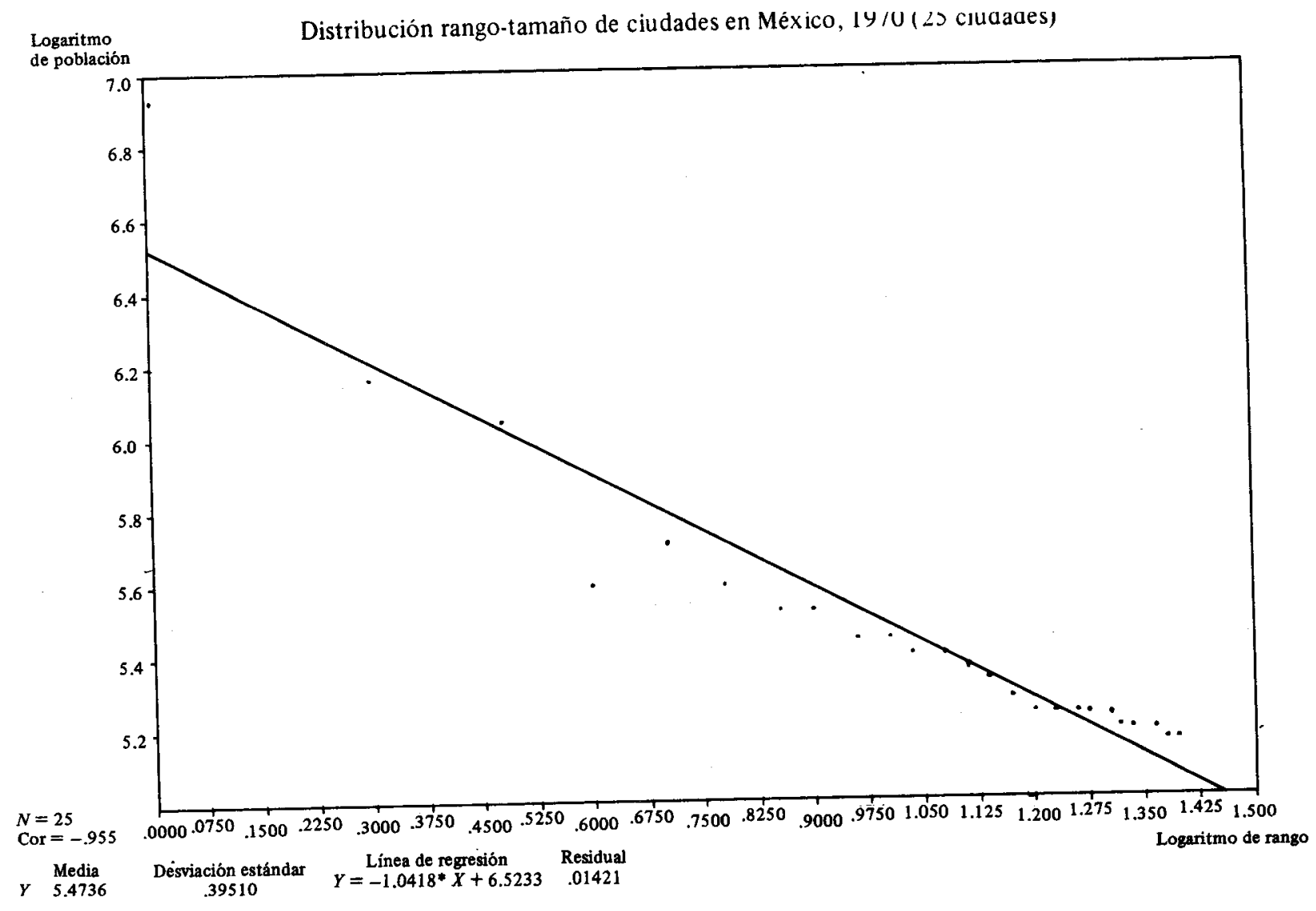


del valor unitario en la misma, indica en el mejor de los casos, la existencia de un impasse en las tendencias de concentración interurbana en el conjunto de ciudades analizadas. Esto significa que, en cierta medida, la dinámica de las 25 ciudades ha contrarrestado al menos el peso concentrador de las tres ciudades más grandes del país. Esta última característica sería la que les conferiría a algunas ciudades el carácter de intermedias bajo un punto de vista estadístico.

\section{Rango-tamaño en las 37 ciudades de mayor tamaño}

Sería incompleta esta última proposición sobre el carácter intermedio del conjunto de ciudades que siguen a las tres más grandes del país, si no se lleva a cabo un análisis complementario sobre la regla rango-tamaño con un mayor número de ciudades, e incluido 1980.

Analizamos la distribución por tamaños para las 37 ciudades más grandes del país entre 1940 y 1980 llevando a cabo un procedimiento equivalente, que también puede constatar una regla rangotamaño y consiste en estimar si el producto rango-tamaño es constante o igual al tamaño de la ciudad más grande -en este caso la ciudad de México- en los años 1940, 1950, 1960, 1970 y 1980. La manera simple ie medir los alejamientos y/o acercamientos a dicha constante, es a través de la desviación estándar existente entre los 37 productos rango-tamaño en cada año considerado.

Obtuvimos cinco valores de desviación estándar, ponderados con el producto rango-tamaño de la ciudad de México, que presentan muy poca diferencia entre sí: de 0.12 para 1940 y 1970 y de 0.11 para 1950 y $1960^{5}$ (véase el cuadro 3).

Estos cinco valores señalan un gran acercamiento al valor que nos daría una constatación exacta de la regla rango-tamaño en cada año (i.e. cero). Nos muestran en forma un poco más precisa que, entre las 37 ciudades más grandes del país, las desviaciones estándar calculadas son muy cercanas a cero.

Nos confirman también la existencia del impasse de concentración interurbana ya que, entre 1940 y 1980, no se observan mayores acercamientos o alejamientos al valor cero de desviación estándar que los que se obtuvieron.

5 Para 1980 hicimos dos estimaciones: tomamos sólo las 25 ciudades para las que tenemos datos del último censo de población y su resultado fue 0.36 , luego incluimos el resto de las ciudades con datos de proyecciones y calculamos 0.12 . 
Brambila/SALAzar: TAMAÑos dE CIUdAdES

Cuadro 3

México: Desviación estándar de los productos rango-tamaño de las 37 áreas urbanas más importantes: 1940 a $1980^{1}$

\begin{tabular}{llllll}
\hline 1940 & 1950 & 1960 & 1970 & $1980^{2}$ & 1980 \\
\hline 0.12 & 0.11 & 0.11 & 0.12 & 0.36 & 0.12 \\
\hline
\end{tabular}

Fuente: Cálculos claborados con base en los datos del cuadro A-1.

1 Se trata de una desviación estándar de los productos rango-tamaño ponderados con el producto rango-tamaño de la ciudad más grande, esto es, la ciudad de México.

2 Esta desviación estándar excluye a las 12 ciudades que no tienen dato censal definitivo en 1980; por lo tanto, se caiculó considerando el producto rango-tamaño de la ciudad de Monterrey como la ciudad de mayor tamaño, con dato censal definitivo, en este año.

\section{Definición de ciudades intermedias}

Aceptar una definición a priori de ciudades intermedias, a partir de las tendencias analizadas sobre el comportamiento rango-tamaño entre 1940 y 1980 , resultaría forzado si no se avanza en el conocimiento de la interdependencia existente entre los tamaños considerados en el conjunto de 37 ciudades.

En este sentido, sería conveniente averiguar si existe una relación estadística y estrecha entre los incrementos de tamaño entre dos rangos dados y el tamaño del rango inferior, según el modelo siguiente que llamaremos función de cambio de tamaño interurbano:

$$
Y=\left(X_{i}-X_{i-1}\right)=a+b X_{i-1}
$$

en donde $X_{i}$ es la población o tamaño de la ciudad de rango $i$ y $X_{i-1}$ es la población o tamaño de la ciudad de rango inmediato inferior $i-1$.

En otras palabras, se trata de encontrar con este modelo una relación proporcional, significativa entre los tamaños de las ciudades consideradas y ordenadas según su rango. 
Los resultados de las funciones estimadas para 1940, 1950, 1960 y 1970, además de haber sido estadísticamente significativos, muestran una tendencia creciente en el coeficiente de correlación entre las variables consideradas, mas no en los incrementos de tamaño de un rango dado y el del rango inmediato superior (parámetro b). Efectivamente, en 1960 disminuye ligeramente el valor del efecto proporcional entre tamaños interurbanos en relación a 1950; en 1980 también da una ligera disminución tanto en el coeficiente de correlación como en el valor del efecto proporcional entre los tamaños urbanos en relación a 1970, pero ambos se mantienen con valores mayores en relación a 1940 y 1960 (véase el cuadro 4).

\section{Cuadro 4}

México: Funciones estimadas del cambio de tamaño interurbano'

\begin{tabular}{lcccc}
\hline \multicolumn{2}{c}{ Valores } & \multicolumn{4}{c}{ Parámetros de análisis de regresión } \\
Añ o & Intersección & $b$ & $r$ & $t_{c}{ }^{2}$ \\
\hline 1980 & -865580 & 3111 & 0.74 & 6.43 \\
1970 & -616824 & 3366 & 0.77 & 7.04 \\
1960 & -345000 & 3087 & 0.73 & 6.23 \\
1950 & -222754 & 3354 & 0.66 & 5.12 \\
1940 & -52419 & 1496 & 0.47 & 3.10 \\
\hline
\end{tabular}

Fuente: Cálculos elaborados con base en datos del cuadro A-2.

1 Función lineal tipo:

$$
y-\left(X_{i}-X_{i-1}\right)=a+b X_{i-1}
$$

en donde $i=37,36,35, \ldots, 1=$ rangos de ciudades en orden decreciente

$X_{i}=$ población de la ciudad en el rango $i$.

$X_{i-1}=$ población de la ciudad en el rango $i-1$.

$r=$ coeficiente de correlación entre $\left(X_{i}-X_{i-1}\right)$ y $X_{i-1}$.

2 Significativas a $95 \%$ de nivel de confianza. 
De esto último, caben dos explicaciones posibles. La primera, buscaría justificar hipotéticamente el comportamiento fluctuante del efecto proporcional entre tamaños urbanos, con el argumento de que sus bajas relativas en 1960 y 1980 son propias de una interacción interurbana, menos propicia a un auge concentrador, por dos razones diferentes: en el primer año por una difusión interurbana del crecimiento económico - vista en términos de cambios en la especialización interurbana- inducida seguramente por la diversificación sectorial del segundo proceso sustitutivo del auge de importaciones en los años sesenta en México y en el segundo año, probablemente por una difusión semejante, pero bajo un contexto de crisis económica con estructuras rígidas a la ampliación del proceso sustitutivo de importaciones, en donde las ciudades más grandes pierden capacidad de atracción en términos relativos de población y de actividades económicas "motoras".

El carácter desconcentrador de la segunda explicación sería mucho más cauteloso, en relación con la primera, ya que si los datos de población del último censo resultan ser mucho mayores en las doce ciudades para las que se utilizaron datos proyectados en 1980, sobre todo en el caso de México y Guadalajara, el coeficiente de correlación y el efecto proporcional entre tamaños urbanos aumentaría, efectivamente en relación a 1970. Esto significaría una mayor interdependencia de tamaños urbanos bajo un esquema de concentración vigente en donde, la importancia dinámica de crecimiento de las ciudades que siguen a las tres primeras en tamaño presentaría un papel paradójico y crucial a la vez: por un lado, la existencia de una dinámica importante de crecimiento e intercambio de rangos propiciaría un comportamiento de distribución de tamaños urbanos muy parecido al rango-tamaño; pero por otro, la interdependencia estrecha entre los tamaños urbanos significaría también el mantenimiento de importantes patrones de concentración de población interurbana, básicamente en las tres ciudades más grandes del país.

En la medida en que los datos definitivos de población de las ciudades de México y Guadalajara -a pesar de llegar a ser mayores que las proyecciones utilizadas en este trabajo- no causen variaciones importantes en el coeficiente de correlación y en el efecto proporcional entre los tamaños urbanos de las 37 ciudades más grandes del país en 1980, y de que los valores definitivos de población de las otras 10 ciudades con rangos inferiores, registren también cifras de población considerablemente mayores en 1980 , se podría verificar una situación en la que se contrarrestaría el peso de las tres ciudades 
principales. De ser así, el papel de la dinámica poblacional de las ciudades que siguen a las tres primeras, dejará de ser paradójico en forma paulatina en los años venideros, en favor del mantenimiento de una distribución de tamaños de ciudades de tipo rango-tamaño.

Sin excluir la posibilidad de que en 1980 aumente el número de ciudades, ubicadas en el tramo de comportamiento tipo rango-tamaño visto gráficamente en apartados anteriores, tales metrópolis merecen la denominación de intermedios por sus importantes efectos en las tres ciudades más grandes y debido a su tendencia a inducir un comportamiento estadístico de distribución de su población tipo rangotamaño, posiblemente también en forma abiertamente desconcentrada en un futuro inmediato en México.

Esto último, de analizarse en términos de sistemas de ciudades, significaría que éstas también serían intermedias, en términos espaciales, por tener latente un potencial inductor de difusión del crecimiento económico, hacia el conjunto de localidades urbanas que parecen seguir un patrón de distribución interurbana de población tipo lognormal (véase su definición en Mills, 1972, pp. 112-115).

\section{ASPECTOS TEÓRICOS DEL PROCESO DE DESCONCENTRACIÓN DE LA POBLACIÓN}

Los resultados presentados en este estudio son básicamente descriptivos porque, como se mencionó en la introducción, es necesario identificar los patrones de distribución de la población urbana antes de afirmar que existe una tendencia hacia la concentración o a la desconcentración demográfica del país. Igualmente, la literatura sobre el desarrollo urbano en México, ha enfatizado en el estudio de los factores que afectan la concentración de la población en aglomeraciones. Sin embargo, no existe una interpretación sistemática y coherente que permita explicar qué factores económicos y demográficos favorecen la. descentralización de la población en diversos centros $y$ de la forma en que se ha observado en México.

En la presente sección se discuten varias interpretaciones teóricas que proporcionan elementos importantes para explicar qué factores favorecen la descentralización, observada en México y para analizar prospectivamente los límites de la concentración de la población en las metrópolis principales del país. 


\section{Desconcentración de una población estacionaria}

En primer lugar, un elemento importante, desde el punto de vista teórico, para explicar la descentralización de la población es la investigación del profesor D. Vining (1977) donde ha demostrado que en una población nacional que crece de una forma estacionaria, la simple reducción en las tasas de mortalidad, favorece un proceso de distribución proporcional de la población. Teóricamente, entonces, una población que reduce su crecimiento paulatinamente, debe tender a distribuirse homogéneamente entre los centros urbanos, de forma que pueda observarse una proporcionalidad entre el crecimiento de las ciudades pequeñas y las grandes.

En el caso de México, la reducción de las tasas de fecundidad, y por lo tanto del crecimiento total de las poblaciones urbanas ha sido observado desde el inicio de los programas de planificación familiar en 1973. El control del crecimiento en la población de la ciudad de México ha mostrado una influencia positiva (en el sentido estadístico) en el crecimiento proporcional de las distintas ciudades en México. Se esperaría teóricamente que, de continuar la reducción de los niveles actuales de crecimiento, el sistema urbano mexicano tendería hacia la proporcionalidad y, consecuentemente, a un decremento de la primacía observada en el país.

La reducción en las tasas de crecimiento afecta la distribución de la población a través de la limitación de la fecundidad en las poblaciones urbanas. En una población cerrada, la reducción de la fecundidad provoca eventualmente la estabilización del crecimiento de todo el sistema, es decir, una situación en la que todas las ciudades crecen al mismo ritmo. Consecuentemente, los sistemas urbanos están sujetos a la misma dinámica de estabilización del crecimiento porque la población inmigrante tiende a adoptar los patrones de fecundidad del lugar de origen de modo que, en un largo plazo, los diferenciales de crecimiento debidos a la fecundidad excesiva de los migrantes, tienden a desaparecer y la dinámica del desarrollo urbano a coincidir con el proceso demográfico de un sistema cerrado.

El hecho de que los migrantes adopten los niveles de fecundidad del lugar de origen ha sido confirmado en distintos países y aparentemente es un fenómeno general, que ocurre con distintos grados de dificultad dependiendo del desfase entre el desarrollo económico y el desarrollo urbano. (Lee, B.S., et al., 1982; Schultz, 1981; Goldstein y Goldstein, 1983; Brambila, 1982). 
Por lo tanto, existen algunos elementos teóricos que explican cómo la dinámica demográfica del país puede, en el mediano y largo plazos, favorecer la desconcentración urbana de las metrópolis principales, en favor del crecimiento proporcional de ciudades pequeñas e intermedias.

Es importante aclarar, sin embargo, que el proceso de desconcentración de la población urbana del país, no implica que su crecimiento se detendrá y mucho menos que la aglomeración de la población en las metrópolis, se reducirá en alguna forma. Tanto el continuo crecimiento como la excesiva aglomeración son factores importantes que explican y favorecen la dinámica económica de estas metrópolis.

\section{Componentes del cambio en las poblaciones urbana y rural}

En segundo lugar, para explicar el proceso de concentración y desconcentración de la población, es necesario considerar la dinámica y perspectivas de los componentes del crecimiento demográfico de tal forma que sea posible determinar las tasas de urbanización y de crecimiento urbano, considerando su relación con la dinámica de la población rural.

Algunos desarrollos teóricos recientes (Rogers, 1972; Ledent, 1981; Ledent, 1982; ONU, 1982) han determinado matemáticamente la relación entre el nivel y las tendencias de la migración rural-urbana basándose en el simple conocimiento de las proporciones de la población que son urbanas y rurales en un tiempo determinado. De acuerdo con las estimaciones de las tasas de migración es posible determinar el porcentaje de crecimiento imputable a migración interna $\mathrm{y}$, a través de métodos proyectivos, se pueden determinar las probabilidades de que tales niveles de migración continuen por un período prolongado.

Básicamente, los resultados de Rogers y Ledent, tanto como los cálculos de la ONU, han demostrado que en México, como en otras regiones del mundo, la tendencia al incremento de la migración ruralurbana, tiene un límite después del cual tiende a disminuir. Para México se estima que ese límite superior fue alcanzado durante la década de los setenta y tenderá a disminuir paulatinamente para alcanzar 10.0 por mil en el año 2000 . Este patrón, lógicamente, no implica que las principales ciudades, tienden a disminuir su tamaño o su crecimiento, sino que la distribución de las poblaciones rural y urbana, se inclina a ser crecientemente más proporcional. 
Las estimaciones de IIASA y de la ONU representan un límite máximo de los componentes del crecimiento demográfico porque no consideran que en el contexto del desarrollo económico de México, el factor económico tendería a desalentar la migración. Este factor es el diferencial de ingresos y bienestar entre el campo y las ciudades y varía en función al volumen y ritmo de crecimiento de la migración. Considerando el diferencial de ingresos y bienestar entre el campo y las ciudades, se esperaría que la creciente migración tendiese a reducir estos diferenciales (porque aumenta el volumen y la composición de la fuerza de trabajo urbano, mientras que disminuye el mercado laboral rural). ${ }^{6} \mathrm{Y}$ consecuentemente tenderá al equilibrio más rápidamente que si sólo se toma en cuenta la dinámica demográfica.

Por lo tanto, desde el punto de vista teórico, es importante considerar que la concentración de la población no puede continuar indefinidamente porque existe un cierto balance demográfico entre las poblaciones rural y urbana $y$, en segundo lugar, porque los diferenciales de ingreso y bienestar tienden necesariamente a equilibrar el sistema demográfico, regulando el volumen de los flujos migratorios. Es entonces, lógico y demostrable empíricamente, que la migración a los grandes centros urbanos depende tanto del deterioro de la situación en el campo, como de la capacidad de los sistemas urbanos para emplear, formal o informalmente, a las poblaciones migrantes.

Aun las condiciones deterioradas de la vida urbana marginal representan una alternativa superior al deterioro rural, sin embargo, en la medida en que la migración aumenta y las posibilidades de vida y de empleo se reducen, es de esperar que se desaliente la migración.

\section{Cambio de tamaño interurbano de tipo logístico}

En tercer lugar, es posible contrastar la estrecha interrelación de tamaños urbanos y sus cambios entre rangos subsecuentes - señalada en el segundo apartado de este trabajo con la ley del efecto proporcional de Yule-Simon. Esta ley establece que, en un sistema de ciudades,

6 Es importante considerar que en este sistema económico existe una tendencia a la reducción del crecimiento de la población rural (vía reducción de la fecundidad), de modo que la oferta de mano de obra en el campo no es infinita. 
la probabilidad del incremento de un habitante a una determinada ciudad es directamente proporcional a su tamaño, $y$, en este contex to, cada ciudad es considerada como una variable aleatoria independiente (Haran y Vining, 1973); mientras que la interrelación de tamaños urbanos implicaría un efecto proporcional entre éstos y, por lo tanto, de carácter no aleatorio.

La ley de Simon prevé una distribución de tamaños de ciudades tipo rango-tamaño cuando se trata de sistemas de población a nivel nacional, en los que el componente rural es importante, $y$ en donde los nacimientos prevalecen sobre las defunciones. El resultado es un predominio abierto de la migración rural-urbana sobre otros tipos de migración. Por el contrario, en sistemas nacionales de población en donde el componente urbano es superior y disminuye la brecha entre nacimientos y defunciones, el patrón de migración por excelencia será el interurbano y se obtendrá una distribución de tamaños cóncava y creciente, en relación a la distribución perfectamente lineal de los logaritmos rango-tamaño.

En este sentido, el caso de las 25 ciudades más grandes en México entre 1940 y 1970 muestra una cierta concavidad no creciente, a juzgar por el número de ciudades que se encuentran distribuidas gráficamente por encima de la recta rango-tamaño ajustada para cada año considerado: 15 en 1940; 10 en 1950; 16 en 1960 y 14 en 1970. Estas tendencias sugieren la existencia probable de un proceso de migración interurbano de importancia creciente, no sólo en virtud de la estrecha interdependencia entre tamaños encontrada entre las 37 ciudades más grandes del país entre 1940 y 1970, sino también por el posible comportamiento log-normal tipo Yule-Simon existente en la distribución del tamaño del resto de localidades urbanas del país, sugerido por los resultados ya analizados en el primer apartado del trabajo.

Si estos argumentos son válidos, los cambios de tamaño interurbanos de ciudades intermedias seguirán estrechamente interrelacionados entre sí, aunque posiblemente bajo una forma logística y en un futuro no inmediato, a juzgar por las pendientes crecientes obtenidas para las funciones lineales de cambio interurbano, estimadas en el segundo apartado, $y$ por los escalonamientos con fuertes reducciones de incrementos de tamaño entre rangos subsecuentes registrados entre 1940 e incluso 1980 (véase cuadro A-2).

Por lo tanto, una tendencia de cambio de tamaño interurbano de tipo logístico en ciudades intermedias, debería ser un aspecto analítico de interés para entender las posibilidades reales de la existencia 
de un proceso de desconcentración urbana en México. Por lo pronto, las mismas funciones lineales de cambio interurbano estimadas no van más allá de sugerir la necesidad de plantear un análisis de semejante tendencia que, con ilustraciones, se representa en la gráfica 10.

\section{Especialización económica interurbana}

En cuarto lugar, podemos considerar la forma en que los procesos de difusión económica territorial -inducidos en la actualidad principalmente por las empresas multinacionales-conllevan cambios importantes en la reorganización de la especialización económica interurbana que modifiquen la alta concentración de población y actividades económicas en el sistema de ciudades del país.

En este sentido, se sabe que en la actualidad la especialización interurbana, como expresión de la división espacial del trabajo, ha sido afectada por la gran movilidad territorial de tales empresas. Movilidad que, a su vez, ha sido producto de la facilidad para descentralizar y desconcentrar especialmente los distintos órganos de gestión y producción, a nivel internacional, en virtud de los factores de localización que se sintetizan primordialmente en una búsqueda generalizada de mercados potenciales de trabajo urbano-industrial, poco calificados y organizados, y que no pertenecen exclusivamente a las ciudades de mayor jerarquía en los distintos ámbitos territoriales de implantación de que se trate: internacional, nacional o regional (Thwaites, 1978).

El efecto de implantación, en términos espaciales, esperado y constatado empíricamente en años recientes ha sido la modificación paulatina, relativa e incluso absoluta de los patrones de difusión territorial jerárquica del crecimiento económico, sobre todo en países avanzados y sumamente industrializados (A. Pred, 1977).

La emergencia creciente de semejantes procesos de difusión económico-territorial no jerárquica en los sistemas de ciudades pueden, por lo tanto, analizarse como resultado de impactos inducidos en la demanda de fuerza de trabajo en mercados regionales; de tal forma que los cambios más evidentes se darían en la modificación misma de los flujos territoriales de empleo y de migración de población, privilegiando flujos de carácter interurbano en relación a los de tipo rural-urbano.

Por esta razón, tales procesos de difusión económico-territorial 


\section{Cuadro A-1}

México: Tamaño de población y rango de las 37 áreas urbanas más importantes del país para 1940, $1950,1960,1970$ y 1980

\begin{tabular}{lrrrrrrrrrrr}
\hline & \multicolumn{3}{c}{1940} & \multicolumn{2}{c}{1950} & \multicolumn{2}{c}{1960} & \multicolumn{2}{c}{1970} & \multicolumn{2}{c}{$1980^{1}$} \\
Ciudad & Tamaño & Rango & Tamaño & Rango & Tamaño & Rango & Tamaño & Rango & Tamaño & Rango \\
\hline 1. México & 1559782 & 1 & 2972334 & 1 & 4909961 & 1 & 8355084 & 1 & $13368315^{*}$ & 1 \\
2. Guadalajara & 240721 & 2 & 401283 & 2 & 811829 & 2 & 1381984 & 2 & $2221053^{*}$ & 2 \\
3. Monterrey & 190128 & 3 & 354114 & 3 & 699263 & 3 & 1095667 & 3 & 1913075 & 3 \\
4. Puebla & 138491 & 4 & 226646 & 4 & 305469 & 4 & 513237 & 4 & 772908 & 4 \\
5. Tampico & 110550 & 5 & 135419 & 7 & 176173 & 9 & 276579 & 9 & 400401 & 12 \\
6. Torreón & 101354 & 6 & 188203 & 5 & 263564 & 5 & 332569 & 8 & 514323 & 7 \\
7. Mérida & 96852 & 7 & 142858 & 6 & 172155 & 11 & 216824 & 14 & 424399 & 9 \\
8. Aguascalientes & 82234 & 8 & 93358 & 12 & 126617 & 15 & 183848 & 15 & 293152 & 18 \\
9. San Luis Potosi & 77161 & 9 & 131715 & 8 & 172332 & 10 & 253638 & 12 & 411544 & 11 \\
10. Orizaba & 76825 & 10 & 86856 & 14 & 108894 & 16 & 139505 & 26 & $170059^{*}$ & 29 \\
11. León & 74155 & 11 & 122726 & 9 & 216246 & 7 & 385817 & 6 & $607996^{*}$ & 6 \\
12. Veracruz & 71720 & 12 & 101246 & 11 & 147501 & 14 & 223136 & 13 & $332131^{*}$ & 15 \\
13. Chihuahua & 56805 & 13 & 87000 & 13 & 158389 & 12 & 262957 & 11 & $412680^{*}$ & 10 \\
14. Pachuca & 53354 & 14 & 58658 & 20 & 66883 & 33 & 86703 & 35 & 110351 & 37 \\
15. Saltillo & 49430 & 15 & 69842 & 15 & 28839 & 19 & 164092 & 20 & 284937 & 12 \\
16. Cd. Juárez & 48881 & 16 & 122566 & 10 & 262119 & 6 & 414908 & 5 & $697291^{*}$ & 5 \\
17. Morelia & 44304 & 17 & 64979 & 17 & 106077 & 17 & 168406 & 18 & 297544 & 16 \\
18. Toluca & 43429 & 18 & 53481 & 18 & 89326 & 23 & 149750 & 23 & $246264^{*}$ & 21 \\
19. Jalapa & 39530 & 12 & 51169 & 23 & 68524 & 31 & 129305 & 27 & $216125^{*}$ & 22 \\
20. Querétaro & 33629 & 20 & 49440 & 25 & 69058 & 30 & 116233 & 31 & 215976 & 23
\end{tabular}




\begin{tabular}{lrlrlrlllll} 
21. Durango & 33412 & 21 & 59869 & 19 & 99436 & 18 & 156507 & 21 & 257915 & 20 \\
22. Irapuato & 32377 & 22 & 49445 & 24 & 83768 & 25 & 118163 & 29 & $172071 *$ & 31 \\
23. Mazatlán & 32117 & 23 & 41754 & 31 & 76874 & 27 & 126325 & 28 & 199830 & 25 \\
24. Oaxaca & 29306 & 24 & 46632 & 27 & 75196 & 28 & 111013 & 32 & $176709 *$ & 30 \\
25. Nuevo Laredo & 28872 & 25 & 57668 & 21 & 92627 & 22 & 152325 & 22 & 201731 & 24 \\
26. Villahermosa & 25114 & 26 & 35418 & 32 & 55360 & 35 & 103694 & 33 & 158216 & 33 \\
27. Celaya & 22766 & 27 & 34424 & 33 & 58851 & 34 & 80638 & 37 & $116903 *$ & 36 \\
28. Culiacán & 22025 & 28 & 48936 & 26 & 85024 & 24 & 172004 & 17 & 340826 & 14 \\
29. Cd. Victoria & 19513 & 29 & 31815 & 35 & 50797 & 37 & 85948 & 36 & 140161 & 35 \\
30. Mexicali & 18755 & 30 & 65749 & 16 & 179539 & 8 & 276167 & 10 & 341559 & 13 \\
31. Hermosillo & 18601 & 31 & 43519 & 29 & 96091 & 21 & 180237 & 16 & 297175 & 17 \\
32. Tepic & 17547 & 32 & 24595 & 37 & 54069 & 36 & 89765 & 34 & 145741 & 34 \\
33. Tijuana & 16486 & 33 & 59952 & 18 & 152473 & 13 & 341067 & 7 & 429500 & 8 \\
34. Matamoros & 15699 & 34 & 45846 & 28 & 97327 & 20 & 140660 & 24 & 188745 & 28 \\
35. Cuernavaca & 14336 & 35 & 43309 & 30 & 77484 & 26 & 167753 & 19 & 192770 & 27 \\
36. Cd. Obregón & 12497 & 36 & 30991 & 36 & 67956 & 32 & 117183 & 30 & 165572 & 32 \\
37. Reynosa & 9412 & 37 & 34087 & 34 & 74140 & 29 & 140480 & 25 & 194693 & 26 \\
\hline
\end{tabular}

Fuente: Unikel, Luis, Gustavo Garza y Crescencio Ruiz Chiapetto, El desarrollo urbano de México: Diagnóstico e implicaciones futuras. México, D. F., El Colegio de México, 2a. edición, 1978 (pp. 158-159), y X Censo General de Población y Vivienda, Secretaría de Programación y Presupuesto, Dirección General de Estadística, 1983. 1 Los datos con asteriscos son estimaciones para 1980 de las áreas urbanas definidas por L. Unikel (ibid. pp. 300-301). El resto de
los datos se refieren a la categoría censal de ciudad y no a áreas urbanas. 
pueden subyacer a toda búsqueda de explicación de las tendencias de concentración y de distribución de tamaños de ciudades analizadas en este trabajo. Asimismo, constituyen una explicación alternativa, distinta a las que tradicionalmente sostienen en todo momento una relación proporcional entre tamaño y especialización urbana (Richardson, 1977). Relación que, a su vez, ha sido constatada en el caso de algunas ciudades intermedias industrializadas y en vías de industrialización en México (Salazar, 1982) y que debe contrastarse con algunos análisis que demuestran, en forma muy sugerente, la coexistencia actual de una marcada especialización económica interurbana con una alta diversificación económica urbana para las 37 ciudades más grandes del país entre 1940 y 1980 (Negrete, 1983).

La razón para llevar a cabo este contraste es que esa coexistencia puede ser manifestación de la vigencia de procesos de difusión económico-territorial no jerárquicos, inducidos por patrones de implantación industrial de grandes empresas móviles multinacionales en el territorio nacional, en conjunción con otras, del sector público y pertenecientes a actividades estratégicas para el desarrollo económico del país en la actualidad.

\section{Conclusiones}

En este ensayo se han analizado diversos aspectos teóricos y empiricos, que permiten entender el proceso de concentración de la población urbana en México. El análisis estadístico de la distribución de los tamaños de ciudades en el país permite concluir que el crecimiento de las grandes y pequeñas urbes en México, debe entenderse como parte de un proceso global de transición entre una sociedad principalmente agrícola, y una sociedad urbana e industrial. A partir de los resultados analizados, es posible inferir que el simple hecho de mostrar cómo la proporción de la población agrícola decrece en favor del crecimiento urbano, o que la proporción de la población urbana que radica en los grandes centros metropolitanos se incrementa, no constituyen una prueba científica satisfactoria de que la población $y$, principalmente la población urbana, tiende a concentrarse a través del tiempo.

De hecho, existen indicios importantes que muestran cómo el crecimiento de las ciudades intermedias en el sistema urbano mexicano, es más dinámico en términos demográficos, si se le compara con las grandes metrópolis. Además, existen evidencias importantes 
de las áreas urhanas más importantes: $1940,1950,19601970$ y $1980^{1}$

\begin{tabular}{|c|c|c|c|c|c|c|c|c|c|c|}
\hline \multirow{2}{*}{$\begin{array}{l}\text { A.io } \\
\text { Rango }\end{array}$} & \multicolumn{2}{|c|}{1980} & \multicolumn{2}{|c|}{1970} & \multicolumn{2}{|c|}{1960} & \multicolumn{2}{|c|}{1950} & \multicolumn{2}{|c|}{1940} \\
\hline & Pob. & $\triangle P o b$. & Pob. & $\triangle P o b$. & $P o b$. & $\triangle P o b$. & Pob. & $\Delta P o b$. & Pob. & $\triangle P o b$ \\
\hline 37 & 110351 & & 80638 & - & 50797 & & 24595 & & 9412 & \\
\hline 36 & 116903 & 6552 & 85948 & 5310 & 54069 & 3272 & 30991 & 6396 & 12497 & 3085 \\
\hline 35 & 140161 & $\begin{array}{r}23258 \\
5580\end{array}$ & $\begin{array}{l}86703 \\
89765\end{array}$ & $\begin{array}{r}755 \\
3062\end{array}$ & $\begin{array}{l}55360 \\
58851\end{array}$ & 1291 & 31815 & 824 & 14336 & 1839 \\
\hline 34 & 145741 & & & 3062 & 58851 & 3491 & 34087 & 2272 & 15622 & 1363 \\
\hline 33 & 158216 & 12475 & 103694 & 13929 & 66883 & 8032 & 34424 & 337 & 16486 & 787 \\
\hline 32 & 165572 & 7356 & 111013 & 7319 & 67956 & 1073 & 35418 & 994 & 17547 & 1061 \\
\hline 31 & 172071 & 6499 & 116233 & 5220 & 68524 & $\begin{array}{c}568 \\
534\end{array}$ & 41754 & 6336 & 18601 & 1054 \\
\hline $\begin{array}{l}30 \\
29\end{array}$ & $\begin{array}{l}176709 \\
178059\end{array}$ & 4638 & $\begin{array}{l}117183 \\
118163\end{array}$ & $\begin{array}{r}950 \\
980\end{array}$ & 69058 & $\begin{array}{r}534 \\
5082\end{array}$ & $\begin{array}{ll}43309 \\
43519\end{array}$ & $\begin{array}{l}1555 \\
210\end{array}$ & 18775 & $\begin{array}{l}174 \\
738\end{array}$ \\
\hline $\begin{array}{l}29 \\
28\end{array}$ & $\begin{array}{l}178059 \\
188745\end{array}$ & $\begin{array}{r}2000 \\
10686\end{array}$ & $\begin{array}{l}118163 \\
126325\end{array}$ & $\begin{array}{r}980 \\
8162\end{array}$ & $\begin{array}{l}74140 \\
75196\end{array}$ & $\begin{array}{l}5082 \\
1056\end{array}$ & $\begin{array}{l}43519 \\
45846\end{array}$ & $\begin{array}{r}210 \\
2327\end{array}$ & $\begin{array}{l}19513 \\
22025\end{array}$ & $\begin{array}{r}738 \\
2512 \\
2512\end{array}$ \\
\hline 27 & 192770 & $\begin{array}{r}10686 \\
4025\end{array}$ & $\begin{array}{l}1263325 \\
129305\end{array}$ & $\begin{array}{l}8162 \\
2980\end{array}$ & $\begin{array}{l}751986 \\
76874\end{array}$ & $\begin{array}{l}1056 \\
1678\end{array}$ & $\begin{array}{l}43846 \\
46632\end{array}$ & $\begin{array}{r}2327 \\
786\end{array}$ & 22766 & $\begin{array}{l}2.312 \\
741\end{array}$ \\
\hline 26 & 194693 & 1923 & 139505 & 10200 & 77484 & 610 & 48936 & 2.304 & 25114 & 2348 \\
\hline 25 & 199830 & 5137 & 14048 & 975 & 83768 & 6284 & & 504 & & 3758 \\
\hline 24 & 201731 & 1901 & 140660 & 180 & 85024 & 1256 & 49445 & 5 & 29306 & 43 \\
\hline 23 & 215976 & 14245 & 149750 & 9090 & 89396 & 4372 & 51169 & 1724 & 32117 & 2811 \\
\hline 22 & 216125 & 149 & 152325 & 2575 & 92627 & 3231 & 53481 & 2312 & 32377 & 260 \\
\hline 21 & 246264 & 30139 & 156507 & 4182 & 96091 & 3464 & 57668 & 4187 & 33412 & 1.03 .5 \\
\hline 20 & 257915 & 11651 & 16405 & 7585 & 97327 & 1236 & & 990 & & 217 \\
\hline 19 & 284937 & 27022 & 1677 & 3661 & 98839 & 1512 & & 1211 & & 5901 \\
\hline 18 & 293152 & 8215 & 168406 & 653 & 99 & 597 & 59952 & & & 3895 \\
\hline 17 & 297175 & 4023 & 172004 & 3598 & 106 & 6641 & & 5027 & 44 & 875 \\
\hline 16 & 297544 & 369 & 180237 & 8233 & 108 & & & 770 & & 4577 \\
\hline 15 & 339131 & 41587 & 183848 & 3611 & 126617 & 17723 & 69 & 4093 & & 549 \\
\hline 14 & $\begin{array}{r}340826 \\
341559\end{array}$ & $\begin{array}{l}1695 \\
733\end{array}$ & 216824 & 32970 & $\begin{array}{l}147501 \\
\end{array}$ & 20884 & 86856 & 170.14 & 53354 & $\begin{array}{l}3924 \\
3451\end{array}$ \\
\hline $\begin{array}{l}13 \\
12\end{array}$ & $\begin{array}{l}341559 \\
400401\end{array}$ & $\begin{array}{r}733 \\
58.842\end{array}$ & $\begin{array}{r}223136 \\
253638\end{array}$ & $\begin{array}{r}6312 \\
30502\end{array}$ & $\begin{array}{l}152473 \\
158389\end{array}$ & $\begin{array}{l}4972 \\
5916\end{array}$ & & & 56805 & $\begin{array}{r}3451 \\
14915\end{array}$ \\
\hline 11 & $\begin{array}{l}400401 \\
411544\end{array}$ & $\begin{array}{l}58842 \\
11143\end{array}$ & $\begin{array}{l}253638 \\
262957\end{array}$ & $\begin{array}{r}30502 \\
9319\end{array}$ & $\begin{array}{l}158389 \\
172155\end{array}$ & $\begin{array}{r}5916 \\
13766\end{array}$ & $\begin{array}{r}93358 \\
101246\end{array}$ & $\begin{array}{l}6358 \\
7888\end{array}$ & $\begin{array}{l}71720 \\
74,55\end{array}$ & $\begin{array}{r}14915 \\
2435\end{array}$ \\
\hline 10 & $\begin{array}{l}411344 \\
412680\end{array}$ & $\begin{array}{r}1345 \\
1136\end{array}$ & 276167 & $1321 \mathrm{C}$ & 172332 & 13177 & $\begin{array}{l}101 \\
122\end{array}$ & 21320 & $\begin{array}{l}7135 \\
76825\end{array}$ & 2670 \\
\hline 9 & 4243 & 11719 & 2765 & 412 & 176173 & 3841 & 122 & 160 & 77161 & 336 \\
\hline 8 & & & 332 & 55990 & 179 & 33 & 131 & & 82234 & 5073 \\
\hline 7 & & & 34 & 8498 & 216 & 367 & & & & 14618 \\
\hline 6 & & & & 4475 & & & & & & 4502 \\
\hline 5 & 6972 & & & 29091 & & & & & & 9196 \\
\hline & 7729 & 75617 & 51 & 98 & & 41 & & & & 279 \\
\hline 3 & 1913075 & 1140167 & 10956 & 582430 & $699 ?$ & 393794 & 354 & 127 & 190 & 51637 \\
\hline 2 & 2221053 & 307978 & $\begin{array}{l}1381984 \\
0.255044\end{array}$ & 286317 & 811829 & 112566 & 401283 & 47169 & 240721 & $\begin{array}{r}50593 \\
319061\end{array}$ \\
\hline 1 & 13368315 & 11147262 & 8355084 & 6573100 & 4509961 & 098132 & 972334 & 571051 & 559782 & 319061 \\
\hline
\end{tabular}

1:i incremento de población se refiere a las diferencias de tamaño entre un rango dado y el inmediato inferior. 
que indican que la dispersión del crecimiento urbano tiende a ser progresivamente más homogénea.

En vista de los hallazgos presentados en este trabajo, ha sido importante elaborar algunos argumentos teóricos que permiten relacionar la dinámica demográfica y el desarrollo económico del país. Principalmente se discute, en el presente artículo, cómo la dinámica del crecimiento demográfico puede favorecer la descentralización del crecimiento urbano, según, las demostraciones matemáticas de $\mathrm{D}$. Vining, de A. Rogers y J. Ledent. De acuerdo con estas elaboraciones teóricas, es posible concluir que el crecimiento urbano está limitado tanto por la estabilidad de su reproducción como por las perspectivas del crecimiento de la migración rural-urbana.

Por otro lado, existen razones de especialización económica que impiden pensar que las metrópolis, en los años siguientes, puedan convertirse en centros únicos de actividad económica.

En conclusión, se han examinado distintos argumentos que son intuitiva y científicamente plausibles, y que permiten interpretar teóricamente la tendencia a la proporcionalidad en el crecimiento de las ciudades en México. Existen evidencias empíricas claras de que no existe una tendencia observable a la concentración de la población urbana del país, sino que las ciudades intermedias y pequeñas muestran una tendencia a crecer proporcionalmente a las grandes metrópolis. En el mejor de los casos, la proporcionalidad en el crecimiento de las ciudades intermedias, con respecto a las grandes metrópolis, ha permanecido constante durante los últimos cuarenta años.

Falta responder, en la argumentación presentada, a una pregunta crucial con respecto a los factores económicos que favorecen la descentralización del crecimiento urbano. La evidencia presentada contradice claramente las interpretaciones que proponen que la concentración urbana puede continuar indefinidamente. Sin embargo, de acuerdo a los argumentos presentados, en México no es posible analizar sistemáticamente cómo se relaciona la dinámica demográfica con el tipo de crecimiento económico dependiente, como se hace en otros países de América Latina y Asia. Queda entonces como una tarea de investigación posterior, analizar bajo qué condiciones es posible el crecimiento homogéneo o proporcional de los sistemas urbanos en una situación de dependencia o en una situación en la que la centralización del capital y de la población es económicamente más eficiente que la descentralización. 
Gráfica 10

Cambio de tamaño interurbano de tipo logístico

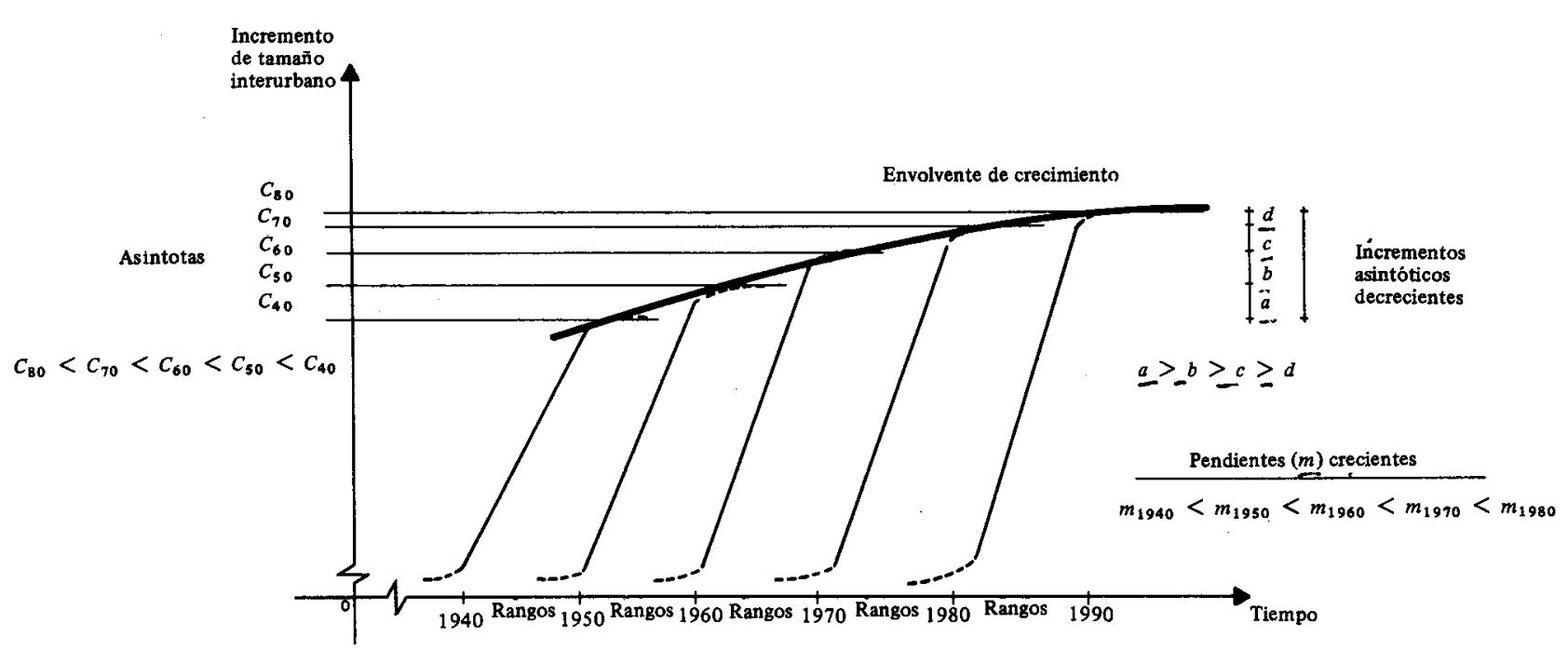


Bibliografía

Brambila, C., Migration and family formation in contemporary Mexico, Universidad de Chicago, 1982 (tesis doctoral).

FaInStein, N. I. y S. S. FAINSTEIN, "Restoration and struggle: Urban policy and social forces" en N. I. Fainstein y S. S. Fainstein (comps.), Urban Policy under Capitalism. Urban Affairs Annual Reviews, vol. 16, Beverly Hills, Sage, 1982.

Goldste IN, S. y A. Goldste IN, Migration and Fertility in Peninsula Malaysia: An Analysis using Life History Data, Reporte Rand N-1860-AID, Santa Mónica, Rand, 1983.

HaRAN, E. G. P. y D. R. VIN ING "Á modified Yule-Simon model allowing for intercity migration and accounting for the observed form of the size distribution of cities", Journal of Regional Science, vol. 13, núm. 3, 1973.

HardoY, J. E., "The building of Latin American cities", en A. Gilbert (comps.), Urbanization in contemporay Latin America, Londres, John Wiley Sons, 1983.

Hymer, S., "Las empresas multinacionales y la ley del desarrollo desigual", El Trimestre Económico, núm. 34, 1980.

LEDENT, JAQUES, "Comparative dynamics of three demographic models of urbanization", Research Report, núms. 80-81, Laxenburg, International form Applied Systems Analysis, 1980.

LEDENT, JAQUES, "Rural-urban migration, urbanization an economic development", en A. Rogers y J. G. Williamson (comps.) Urbanization and Development in the Third World, Laxenburg, International Institute for Applied Systems Analysis, 1982.

Lek, B. S., A. M. JAmal y M. V. E. Rulison, The influence of rural-urban migration of the fertility of migrants in developing countries: analysis of Korean data, First Report, Louisiana, Research Triangle Institute, 1981.

Mills, E., Urban Economics, Illinois, Scott, Foresman and Company, 1972.

Negrete, MA., E., "Especialización y diversificación urbana en México (1940-1980)", ponencia presentada en el coloquio El desarrollo urbano de México: Problemas y perspectivas, Programa Universitario Justo Sierra, Universidad Nacional Autónoma de México, 30 de octubre de 1983 (mimeog.).

Organización de las Naciones UnIDAS, Modalidades del crecimiento de la población urbana y rural, Nueva York, Naciones Unidas, 1981.

PARR, J. B., "A class of deviations from rank-size regularity: three interpretations", Regional Studies, vol. 10, 1976. 
Pred, A., City Systems in Advanced Economies, Londres, Hutchinson, 1977.

RichaRDSON, H. W., "Theory of the distribution of city sizes: Review and prospects", Regional Studies, vol. 7, 1973. -, "City size and national spatial strategies in development countries", Worl Bank Staff, Working Paper, núm. 252, April 1977.

ROGERS, A., "Sources of urban population growth and urbanization, 1950-2000: A demographic accounting", en A. Rogers y J. G. Williamson (comps.). Urbanization and Development in the Third World, op. cit.

ROSEN, K. T. y M. RESNICK, "The size distribution of cities: An examination of the Pareto law and primacy", Journal of Urban Economic, vol. 8, 1980.

Salazar, H., Dinámica de crecimiento de ciudades intermedias en México: los casos de León, San Luis Potosi y Torreón 1970 1980, El Colegio de México y Consejo Nacional de Población (en prensa).

THwaIres, A. T., "Technological change, mobile plants and regional development", Regional Studies, vol. 12, 1978.

VINING, D. R., "The rank-size rule in the obsence of growth", Journal of Urban Economic, vol. 4, 1977.

UN IKEL, L., G. GARZA y C. RUiz ChIA PETTO, El desarrollo urbano de México: Diagnóstico e implicaciones futuras, México, $\mathrm{El} \mathrm{Co-}$ legio de México, 2a. edición, 1978. 